\title{
Cosmic Ray transport in the presence of a CR-driven galactic wind
}

\author{
S. Recchia* \\ GSSI \\ E-mail: sarah.recchialgssi.infn.it \\ P. Blasi \\ INAF, GSSI \\ E-mail: blasiearcetri.astro.it

\section{G. Morlino} \\ GSSI \\ E-mail: giovanni.morlino@gssi.infn.it
}

The transport of cosmic rays (CRs) in the Galaxy is known to be affected by the possible presence of winds launched from the Galactic disc. When these winds are pre-assigned, with a given velocity it can be checked that the effects on transport are limited to energies below $\sim 10 \mathrm{GeV}$. However, CRs escaping from the Galaxy can generate a galactic wind. The hydrodynamics of such wind has been studied in the past, showing that stationary outflow solutions to the wind equation exist for a wide range of the input parameters.

Here we discuss a semi-analytical kinetic calculation for solving the CR transport problem coupled to the hydrodynamic problem of CR-driven galactic winds. In our nonlinear approach the CR diffusion coefficient is due only to scattering on self-generated waves. Our computation allows a self-consistent determination of the CR spectrum and diffusion coefficient, as well as the characteristics of the wind itself.

The 34th International Cosmic Ray Conference,

30 July- 6 August, 2015

The Hague, The Netherlands

${ }^{*}$ Speaker. 


\section{A physical model for CR-driven Galactic winds}

The observed secular escape of CRs from the Galaxy poses the question of whether the CR pressure gradient in the Galaxy may launch winds. This issue has been addressed by several authors (see for instance [1],[2], [9] and [10]) and the result of their investigation can be summarized as follows: in order for a galactic wind to be launched against the galactic gravitational force solely due to the gas pressure gradients, quite high (order of $\sim 10^{8}-10^{9} \mathrm{~K}$ ) temperature for the gas is required, much higher than the temperature of the standard ISM. Thus, purely thermally driven winds can be expected to occur mainly in galaxies with an active nucleus or in starburst galaxies with very high temperature gas in their ISM.

However, if we also take into account CRs (and Alfvén waves) pressure gradients, winds can be easily launched without any restricting requirement on the gas temperature or on other physical parameters involved in the calculation (gas density and pressure, CR and wave pressure, background magnetic field and the gravitational potential).

A thorough study of the hydrodynamics of Galactic winds, which includes both CR and Alfvén waves in the wind dynamics, is provided by Breitschwerdt et al. in [1]. Their basic idea is that supernova ( $\mathrm{SN}$ ) explosions in the galactic disk may create magnetic flux tubes perpendicular to the galactic disk (as shown in Fig. 1) in which CRs escape from the galaxy. In this scenario CRs would propagate in a parallel diffusion regime and CR streaming instability can play a role. In general CR propagation in the flux tube will be a superposition of convection, wave drift and diffusion, and which propagation mechanism dominates depends on the characteristics of the halo.

Near the disk $\left(z_{0} \lesssim 300 p c\right)$ the ISM is expected to be only partially ionized so that waves are dumped by ion-neutral friction and the coupling between ISM and CRs is weak. In this region CR propagation is expected to be diffusion-dominated.

In the halo $\left(z_{0} \gtrsim 300 p c\right.$ above the galactic disk) the ISM is expected to be nearly completely ionized ${ }^{1}$ and the CR pressure gradients large, so that wave production due to CR streaming instability is expected to be efficient enough to keep CRs well coupled to the ISM. In this region the wind can be launched and CR propagation is expected to be advection-dominated.

In [1] only the region $|z| \gtrsim z_{0} \simeq 300 \mathrm{pc}$ is considered and the coupling between CRs and ISM is assumed ideal (i.e diffusion is neglected). Under these assumptions the authors solve the stationary wind equation for a given set of gas density and pressure, CR and wave pressures and background magnetic field at $z_{0}$, where the wind is launched. The computation, which includes the galactic and Dark Matter gravitational potential, is performed in the flux-tube geometry shown in Fig. 1, and returns a wind profile (gas velocity profile) which starts as subsonic and becomes supersonic as in a De Laval nozzle problem. The result of the computation is the spatial profile of the gas density, of the gas and Alfvén wave velocity and of the CR and wave pressures. A typical velocity profile obtained with this calculation is shown in Fig. 2.

\footnotetext{
${ }^{1}$ We note that the presence of diffuse clouds above the disk has been detected (Wakker, B. P. 2001, ApJS, 136, 463) but the expected filling factor is small, hence we do not expect these clouds to significantly affect the coupling between CRs and Alfvén waves.
} 


$$
\begin{aligned}
& A(z)=1+\left(\frac{z}{Z_{0}}\right)^{2} \\
& Z_{0} \sim 15 \mathrm{kpc}
\end{aligned}
$$

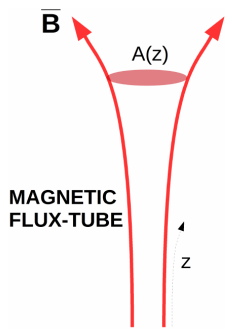

GALACTIC DISK

Figure 1: Magnetic flux-tube geometry.

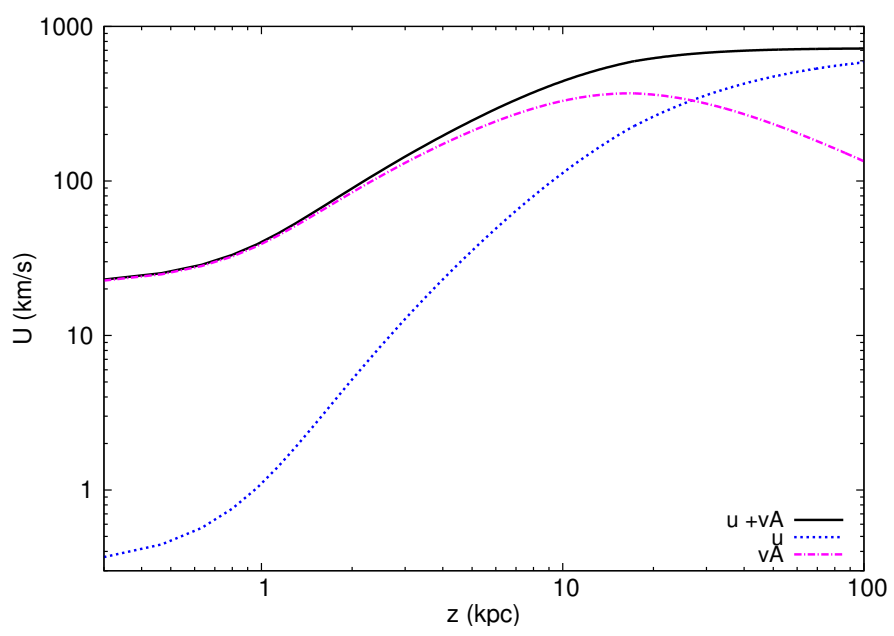

Figure 2: Advection velocity profile derived from the hydrodynamic calculation with input parameters at the wind base: $R_{0}$ (galactocentric distance $)=8 \mathrm{kpc}$, $z_{0}($ wind base $)=300 \mathrm{pc}, \rho_{0}($ gas density $)=16.7 \mathrm{e}-27 \mathrm{~g} / \mathrm{cm}^{3}, P_{g 0}($ gas pressure $)=2.8 \mathrm{e}-$ $13 \mathrm{erg} / \mathrm{cm}^{3}, P_{C R 0}($ CR pressure $)=3 \mathrm{e}-13 \mathrm{erg} / \mathrm{cm}^{3}, P_{w 0}(\mathrm{CR}$ pressure $)=4 \mathrm{e}-16 \mathrm{erg} / \mathrm{cm}^{3}$, $B_{0}$ (background magnetic field $)=1 \mu \mathrm{G}$. Here wave damping has not been included and the Navarro-Frenk-White Dark Matter potential has been used. The resulting velocity profile has $u_{0} \sim 0.3 \mathrm{~km} / \mathrm{s}$ and $v_{A 0} \sim 22 \mathrm{~km} / \mathrm{s}$.

\section{A qualitative picture of the transport of CRs in a CR-driven galactic wind}

The problem of CR transport in a CR-driven galactic wind has been studied in previous work (see for instance [3]). In their approach they focus on the region $z_{0} \gtrsim 300 p c$, where the CR streaming instability is expected to be efficient, the coupling between CRs and ISM strong and the wind has been launched as illustrated in [1]. In this region they also assume that the CR streaming instability is locally balanced by nonlinear Landau damping. The equilibrium wave spectrum is determined by equating the growth rate and damping rate of these two processes. This also provides the CR self-generated diffusion coefficient.

The authors look for an approximated analytic solution to the $\mathrm{CR}$ transport equation under the assumption (justified a posteriori) that $\mathrm{CR}$ diffusion dominates over advection up to a characteristic distance $s^{\star}(p)$ which depends on the particle momentum. Above that distance advection dominates. $s^{\star}(p)$ can be estimated by equating the diffusion and the advection time, i.e.

$$
\frac{\left(s^{\star}(p)\right)^{2}}{D(z, p)}=\frac{s^{\star}(p)}{u(z)+v_{A}(z)},
$$

where $u(z)$ and $v_{A}(z)$ are the wind velocity and the Alfvén speed, respectively. In this scenario the diffusion-advection boundary $s^{\star}(p)$ behaves as an effective boundary for particles of momentum $p$ in a "leaky-box" model. In the traditional "leaky-box" model the CR spectrum at the disk is given by $f_{0}(p)=\frac{Q_{0}(p)}{2 \pi R_{d}{ }^{2} H} \frac{H^{2}}{D}$, where $Q_{0}$ is the injection spectrum, $D$ the diffusion coefficient and $R_{d}$ the galactic disk radius. $H$ is the location of the free escape boundary, an artificial boundary where the $\mathrm{CR}$ distribution function is set to zero in order to guarantee the stationarity of the propagation problem and is usually taken to be of order $\simeq 4 \mathrm{kpc}$.

In this approach $H$ is replaced by $s^{\star}(p)$, which is momentum-dependent and does not need to be 
artificially imposed but derives self-consistently from the solution of the transport problem. The CR distribution function at the disk thus becomes

$$
f_{0}(p)=\frac{Q(p)}{2 \pi R_{d}^{2} s^{\star}(p)} \frac{s^{\star^{2}}(p)}{D} .
$$

Typical scaling with momentum of $f_{0}(p), s^{\star}(p)$ and $D(p)$ are then deduced by the authors under the assumption that the wind velocity profile is

$$
u(z)+v_{A}(z)=\left\{\begin{array}{lc}
u_{0} \frac{z}{s_{0}} & z<Z_{0}(\sim 15 \mathrm{kpc}) \\
\text { const } & z>Z_{0}(\sim 15 \mathrm{kpc})
\end{array}\right.
$$

where $Z_{0}$ is the length scale over which the flux tube starts opening up (Fig. 1), $u_{0} \sim 100 \mathrm{~km} / \mathrm{s}$ and $s_{0} \sim 3 \mathrm{kpc}:$

$$
\begin{gathered}
D(p) \sim p^{\gamma-3} \\
s^{\star}(p) \sim \begin{cases}\sqrt{D(p)}=p^{\frac{\gamma-3}{2}} & z<Z_{0} \\
\sqrt[3]{D(p)}=p^{\frac{\gamma-3}{3}} & z>Z_{0}\end{cases} \\
f_{0}(p) \sim \begin{cases}\frac{Q_{0}(p)}{\sqrt{D(p)}}=p^{-\frac{3}{2}}(\gamma-1) & z<Z_{0} \\
\frac{Q_{0}(p)}{D(p)^{2 / 3}}=p^{\frac{-5 \gamma+6}{3}} & z>Z_{0} .\end{cases}
\end{gathered}
$$

This simple model shows how $s^{\star}$ moves away from the disk as $p$ increases and predicts a spectral steepening at the momentum for which $s^{\star}(p) \sim Z_{0}$. For the values of advection speed and diffusion coefficient used in [3], a momentum of $10 \mathrm{GeV}$ corresponds to $s^{\star} \sim 1 \mathrm{kpc}$ and $1 \mathrm{TeV}$ to $s^{\star} \sim Z_{0}=$ $15 \mathrm{kpc}$.

\section{A semi-analytical approach to the CR transport equation coupled to the CR-driven wind equation}

Here we discuss a semi-analytical kinetic approach to solving the CR transport equation coupled to the hydrodynamic equations of a CR-driven wind. Our procedure can be summarized as follows: we solve the hydrodynamic problem for a given set of the input parameters at the wind base $z_{0}$, using the method discussed in [1]; for $z>z_{0}$ above the disk we obtain the advection velocity spatial profile $U(z)=u(z)+v_{A}(z)$ (gas plus Alfvén speed), the CR pressure profile $P_{c r}(z)$ and the wave pressure profile $P_{w}(z)$; then we use the obtained advection velocity in the CR transport equation and we recursively solve the equation in integral form, as we are going to show in the remaining of this section. The output of this computation is the CR spectrum and diffusion coefficient and the wave spectrum. From the CR and wave spectrum we can deduce an updated CR and wave pressure profile which we can feedback into the hydrodynamic computation and start a new iteration cycle. The method converges to the solution of the problem when the CR and wave pressure profiles derived from the hydrodynamic calculation coincide with those derived from the kinetic calculation. 


\subsection{Method for solving the transport equation}

The stationary transport equation of CRs in three spatial dimensions reads

$$
\vec{\nabla} \cdot[D \vec{\nabla} f]-\vec{U} \cdot \vec{\nabla} f+\vec{\nabla} \cdot \vec{U} \frac{1}{3} \frac{\partial f}{\partial \ln p}+Q=0 .
$$

The calculation is carried out in the flux-tube geometry shown in Fig. 1. Expressing all quantities in terms of the curvilinear coordinate along the magnetic field lines $z$ and applying the prescription for the gradient and divergence operators in the flux tube (see [1]),

$$
\begin{aligned}
& \vec{\nabla} S=\frac{d S}{d z} \hat{z} \\
& \vec{\nabla} \cdot \vec{V}=\frac{1}{A(z)} \frac{d}{d z}[\vec{A}(z) \cdot \vec{V}],
\end{aligned}
$$

the transport equation becomes one-dimensional

$$
\frac{\partial}{\partial z}\left[A(z) D(z, p) \frac{\partial f(z, p)}{\partial z}\right]-\left[A(z) U(z) \frac{\partial f(z, p)}{\partial z}\right]+\frac{d[A(z) U(z)]}{d z} \frac{1}{3} \frac{\partial f(z, p)}{\partial \ln p}+A(z) Q(z, p)=0 .
$$

Here $A(z)$ is the flux-tube area and $U(z)=u(z)+v_{A}(z)$ derives from the hydrodynamic calculation. The wave spectrum $\mathfrak{F}(z, p)$, and the diffusion coefficient $D(z, p)$, are obtained by requiring the local balance between the growth of the CR streaming instability and the nonlinear Landau damping, i.e by equating the growth rate and damping rate of these two processes [4]

$$
\begin{aligned}
& \Gamma_{C R}=\frac{16 \pi^{2}}{3} \frac{v_{A}}{\mathfrak{F} B^{2}}\left[p^{4} v(p)\left|\frac{\partial f}{\partial z}\right|\right]_{p=p_{\text {res }}} \\
& \Gamma_{D}=\left(2 c_{k}\right)^{-3 / 2} k v_{A} \mathfrak{F}^{1 / 2}
\end{aligned}
$$

from which we get

$$
\begin{gathered}
\mathfrak{F}(z, p)=2 c_{k}\left[\frac{p^{4} v(p)\left|\frac{\partial f}{\partial z}\right| \frac{16 \pi^{2}}{3} r_{L}(z, p)}{B^{2}(z)}\right]^{2 / 3} \\
D(z, p)=\left.\frac{1}{3} \frac{v(p) r_{L}(z, p)}{\mathfrak{F}}\right|_{k=1 / r_{L}\left(p_{\text {res }}\right)} .
\end{gathered}
$$

Let us note that Eq. 3.4 is highly nonlinear because both the diffusion coefficient and the advection velocity depend on the CR distribution function.

We look for an implicit solution of the transport equation 3.4 with and injection term of the type $Q(z, p)=Q_{0}(p) \delta(z)$. We integrate the equation in $(z, H)$ with boundary condition $f(H, p)=0$ and around the disk $\left(0^{-}, 0^{+}\right)$with boundary condition $f(0, p)=f_{0}(p)$. The outer boundary $H$ here is set just for numerical purposes, and can be also located at spatial infinity. The result is

$$
f(z, p)=\int_{z}^{H} \frac{d z^{\prime}}{A\left(z^{\prime}\right) D\left(z^{\prime}, p\right)} G\left(z^{\prime}, p\right) \exp ^{-\int_{z}^{z^{\prime}} d z^{\prime \prime} \frac{U\left(z^{\prime \prime}\right)}{D\left(z^{\prime \prime}, p\right)}},
$$

where

$$
G(z, p)=\frac{A_{0} Q_{0}(p)}{2}+A_{0} U_{0} f_{0}(p) \bar{q}_{0}-\int_{0}^{z} d z^{\prime} \frac{d(A U)}{d z^{\prime}} \frac{f}{3} q
$$




$$
\begin{gathered}
q(z, p)=-\frac{\partial \ln \left(f p^{3}\right)}{\partial \ln p} \\
\bar{q}_{0}(p)=1-\frac{1}{3} \frac{\partial \ln f_{0}(p)}{\partial \ln p} .
\end{gathered}
$$

The non-linearity of the problem is "hidden" in the function $G(z, p)$. The solution of such equation, given an advection velocity profile, is computed recursively starting from a guess on the function $f(z, p)$.

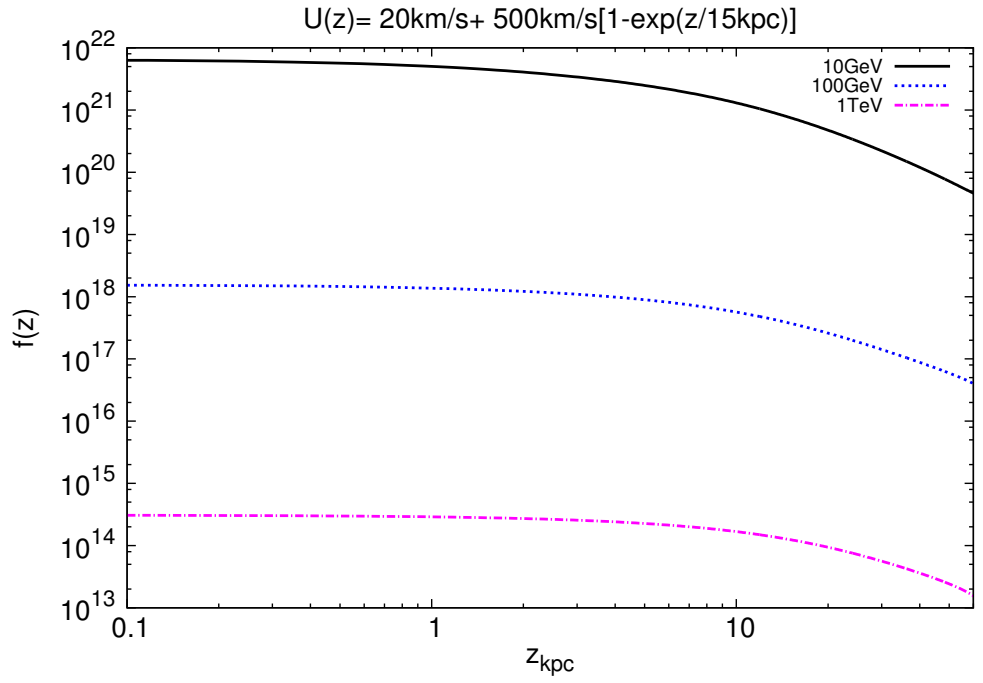

Figure 3: Spatial CR distribution function derived with wind profile which is $20 \mathrm{~km} / \mathrm{s}$ at the disk, then grows linearly up to $Z_{0}$ (where the flux-tube starts opening up) and then becomes flat. The result is shown at $10 \mathrm{GeV}, 100 \mathrm{GeV}$ and $1 \mathrm{TeV}$.

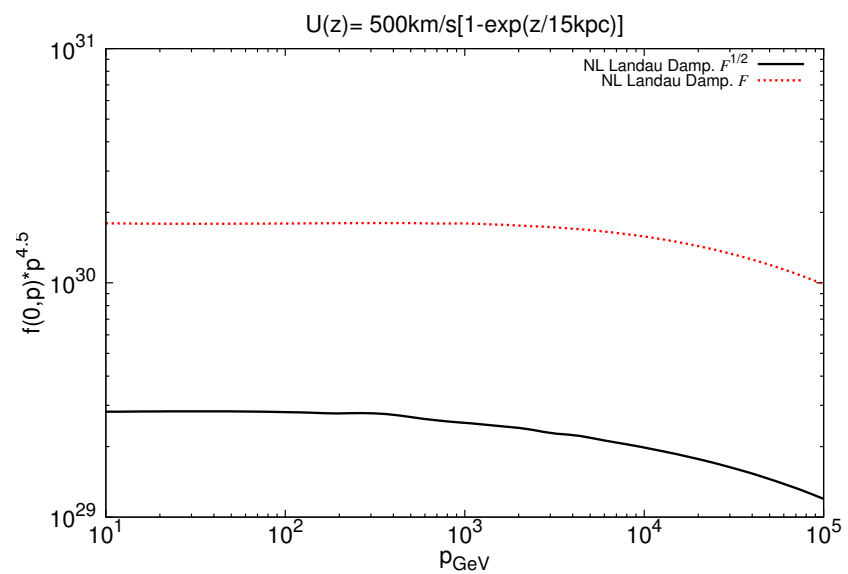

Figure 4: $C R$ spectrum derived with wind profile which vanishes at the disk, then grows linearly up to $Z_{0}$ (where the flux-tube starts opening up) and then becomes flat. The result is shown for two expressions of nonlinear Landau damping. Note the spectral steepening at $\sim 1 \mathrm{TeV}$ due to the opening of the flux-tube.

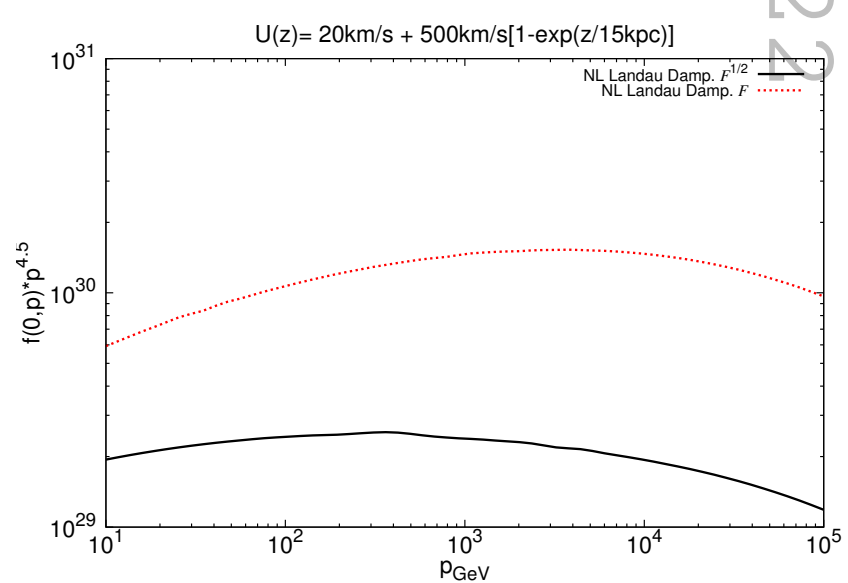

Figure 5: CR spectrum derived with wind profile which is $20 \mathrm{~km} / \mathrm{s}$ at the disk, then grows linearly up to $Z_{0}$ (where the flux-tube starts opening up) and then becomes flat. The result is shown for two expressions of nonlinear Landau damping. Note the low energy cut-off due to the non-zero velocity at the disk. 


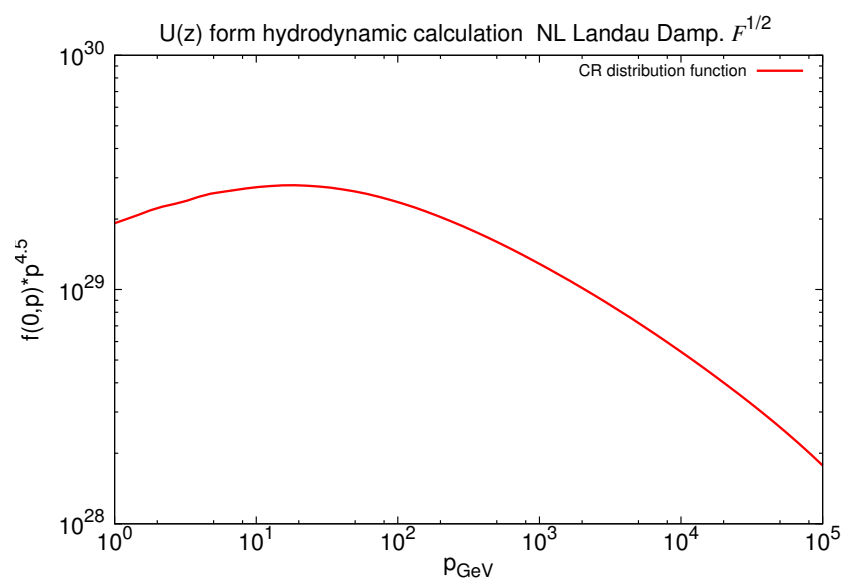

Figure 6: CR spectrum obtained with the velocity profile of Fig. 2. The computation has been done by iterating on both the kinetic and hydrodynamic calculations.

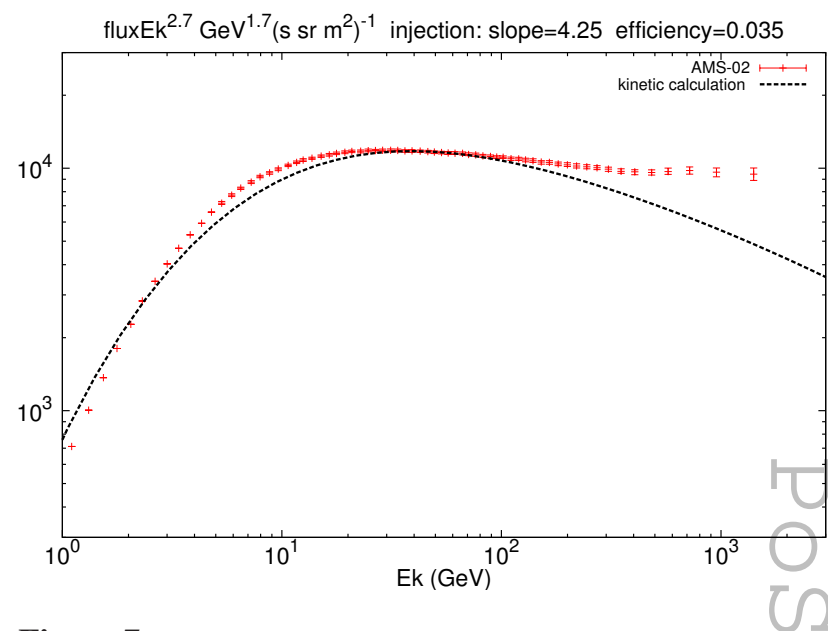

Figure 7: Rough comparison between the AMS-02 data and the spectrum of Fig. 6. The injection spectrum has slope 4.25 and injection efficiency $3.5 \%$. Solar modulation has been applied with $\Phi=500 M V$.

\subsection{Preliminary results}

In this section we show some preliminary results of our method. In Fig. 4 and 5 we show the CR spectrum for an advection velocity profile which grows linearly up to $Z_{0}$ (where the flux-tube starts opening up) and then becomes flat. The valocity at the disk is respectively zero and $20 \mathrm{~km} / \mathrm{s}$. In the literature two different expressions for the non linear Lanadau damping can be found (see [4] for Eq. 3.13 and [3] for Eq. 3.14)

$$
\begin{aligned}
\Gamma_{D} & =\left(2 c_{k}\right)^{-3 / 2} k v_{A} \mathfrak{F}^{1 / 2} \quad \text { later used in our calculation } \\
\Gamma_{D} & =k v_{A} \mathfrak{F}
\end{aligned}
$$

here we show the result for both expressions. In both cases we have a spectral steepening due to the geometry of the diverging flux-tube. The way the spectral slope changes depends on the type of damping, which changes the diffusion coefficient. A non zero base velocity induces a low-energy cut-off due to the fact that low energy particles get advected.

In Fig. 3 we show the spatial dependence of the CR distribution function at energies $10 \mathrm{GeV}$, $100 \mathrm{GeV}$ and $1 \mathrm{TeV}$ for the same advection velocity as in Fig. 5. The CR distribution function decreases slowly (linearly, as in the "leaky-box" model) in z up to a characteristic distance which corresponds to $s^{\star}$. Then it decreases much faster. As expected, $s^{\star}$ is momentum-dependent and increases with momentum.

In Fig. 6 we show the result of our method when the starting velocity profile is the one shown in Fig. 2. The injection spectrum is assumed to be a power-law with index 4.25 and injection efficiency $3.5 \%$. Solar modulation has been taken into account, with modulation potential $\Phi=500 \mathrm{MV}$ (see [8]). The obtained spectrum is shown together with the AMS-02 ([7]) data in Fig. 7 and it roughly reproduces the AMS data for energies $\lesssim 100 \mathrm{GeV}$. As expected, the observed spectral hardening at higher energies cannot be reproduced with the assumed model, which instead predicts a spectral steepening and does not take into account any pre-existing turbulence ([5], [6]). 


\section{Conclusions}

We developed a method for solving self consistently the CR transport equation coupled to the CR-driven galactic wind hydrodynamic equations. In our non-linear approach both the CR diffusion coefficient and the wind profile are generated by CRs. We showed that the momentum dependence of the CR spectrum is determined by the characteristic distance $s^{\star}(p)$ where the propagation from diffusion-dominated becomes advection-dominated. This distance increases with momentum and can be roughly seen as a momentum-dependent boundary for a "leaky-box" model. Finally we compared the spectrum obtained with a realistic velocity profile, derived from the hydrodynamic calculation, with the AMS-02 data and showed that, with and injection efficiency of 3-4\%, we can roughly reproduce the data below $\lesssim 100 \mathrm{GeV}$.

Our calculation can be generalized by taking into account the rotation of the galaxy and the CR diffusion term in the hydrodynamic computation (see [9]). In future work, we intend to use the computation presented here and its generalizations for extensively investigating the phenomenological implications of CR-driven Galactic winds.

\section{References}

[1] D. Breitschwerdt, J.F. McKenzie, H.J. Völk, I. Cosmic ray and wave-driven winds from the Galaxy, Astron. Astrophys. 245 (79-98) 1991

[2] F. Ipavich, Galactic winds driven by cosmic rays, The Astrophysical Journal 196 (107-120) 1975

[3] V.S. Ptuskin, H.J. Völk, V.N. Zirakashvili, D. Breitschwerdt, Transport of relativistic nucleons in a galactic wind driven by cosmic rays, Astron. Astrophys. 321 (434-443) 1997

[4] P. Blasi, E. Amato and P.D. Serpico, Spectral breaks as a signature of cosmic ray induced turbulence in the Galaxy, LAPTH- 0242012 [arXiv: 1207 . 3706v1]

[5] R. Aloisio and P. Blasi, Propagation of galactic cosmic rays in the presence of self-generated turbulence, JCAP 1307 (2013) 001 [arXiv: 1306.2018 ]

[6] R. Aloisio, P. Blasi and P.D. Serpico, Non-linear cosmic ray Galactic transport in the light of AMS-02 and Voyager data, [arXiv:1507.00594 [astro-ph.HE]]

[7] AMS-02 Collaboration, [http://www.ams02.org/]

[8] G. Di Bernardo, C. Evoli, D. Gaggero, D. Grasso and L. Maccione, Unified interpretation of cosmic-ray nuclei and antiproton recent measurements, [arXiv:0909.4548v4 [astro-ph. HE]]

[9] D. Breitschwerdt, J.F. McKenzie, H.J. Völk, II. Rôle of the disk-halo interface in cosmic ray driven galactic winds, Astron. Astrophys. 269 (54-66) 1993

[10] D. Breitschwerdt and E.A. Dorfi, Time-dependent galactic winds. I. Structure and evolution of galactic outflows accompanied by cosmic ray acceleration, [arXiv: $1304.1311 \mathrm{v}$ ] [astro-ph. HE] ] 